\title{
Breath Figure Patterns in the Oxidation of Boron Nitride
}

\author{
Thanh $\mathrm{Ba} \mathrm{Do}^{\dagger}$ and John W. Halloran \\ Department of Materials Science and Engineering, University of Michigan, Ann Arbor, Michigan 48109-2136
}

\begin{abstract}
Liquids condensing on solids can form a pattern known as "breath figures." Here we report similar patterns for liquid boron oxide droplets formed by high temperature oxidation reaction of boron nitride. Boron oxide does not wet boron nitride, so the liquid $\mathrm{B}_{2} \mathrm{O}_{3}$ oxide forms small droplets. As oxidation proceeds at $1200^{\circ} \mathrm{C}$, the oxide created by reaction on the surface migrates to existing droplets or forms new droplets. The average diameter and maximum diameter of the boron oxide droplets increase with time following the kinetics of breath figures, similar to water condensation breath figures for dew drops.
\end{abstract}

\section{Introduction}

$\mathrm{V}$ APOR can condense on cold surfaces in a self-similar pattern of liquid droplets known as "breath figures". Rayleigh described how droplets size increases during condensation by a combination of growth and coalescence. ${ }^{1}$ Beysens and Knopler $^{2}$ showed that, as condensation deposits additional liquid, the average droplet size $\langle D\rangle$ increases with time according to scaling laws $\langle D\rangle \sim t^{\alpha}$, where the scale parameter depends on area fraction coverage, wetting angle, and other factors. The maximum droplet size $D_{\max }$ increases in a characteristic stepwise fashion, ${ }^{3}$ with the steps associated with coalescence events. Breath figures have received a great deal of attention in a variety of areas, including self-assembled bubble arrays, ${ }^{4}$ dew drops, ${ }^{5}$ lithography masks, ${ }^{6}$ and templated films. ${ }^{7}$ We find similar droplet patterns in liquid boron oxide formed on the surface of boron nitride by high temperature oxidation. In this case, the liquid forms not by condensation from a vapor, but by reaction of the substrate with oxygen. This could be relevant to the oxidation of $\mathrm{BN}$ or other boria-formers, because it shows that the boria may not be a continuous film, but rather a pattern of isolated droplets.

Boron oxide is a liquid above $460^{\circ} \mathrm{C}$, with a significant volatility at temperatures above $800^{\circ} \mathrm{C}$, where boron nitride oxidation is rapid. ${ }^{8}$ Boron nitride is poorly wetted by $\mathrm{B}_{2} \mathrm{O}_{3}$. As oxidation proceeds, the BN surface becomes decorated by droplets of liquid oxide. Figure 1 shows the surface of a hot-pressed $\mathrm{BN}$ coupon exposed to dry air for $8 \mathrm{~h}$ at $1200^{\circ} \mathrm{C}$. The droplet features are glassy $\mathrm{B}_{2} \mathrm{O}_{3}$ at room temperature. These glassy $\mathrm{B}_{2} \mathrm{O}_{3}$ droplets can only be observed if the sample is carefully isolated from humidity, which otherwise would quickly create artifacts by hydration. The hydrated oxide observed after exposure to moist air is quite different and does not represent the actual oxide formed at high temperature. Our samples were reacted and stored in a special apparatus to assure a water-free environment, ${ }^{9}$ which preserved the pristine $\mathrm{B}_{2} \mathrm{O}_{3}$ droplets.

At the oxidation temperature, the reaction forms a now-wetting $\mathrm{B}_{2} \mathrm{O}_{3}$ liquid. Breath figures are seen when non-wetting liquids form by condensation, so it is interesting to determine if

D. Butt—contributing editor reaction-formed liquids might also display breath figure kinetics. We examined $\mathrm{B}_{2} \mathrm{O}_{3}$ droplet growth to determine if it could be represented with breath figure growth kinetics. We measured the size of the all the visible droplets on micrographs from on BN samples oxidized for $0.5-30 \mathrm{~h}$. The average droplet size vs. oxidation time is shown as a log-log plot in Fig. 2 (the size of the symbols are larger than the approximately $10 \%$ variation of the average droplet size for each oxidation time). A linear regression line drawn through the average diameters was fit to $\langle D\rangle\rangle \sim t^{\alpha}$, with a scaling exponent $\alpha=0.90+/-0.06$. A scaling exponent of $\alpha=1$ is expected for the coalescence-dominated stage of liquid droplets on a liquid substrate. ${ }^{10}$ During coalescence growth, droplets grow by flow of liquid from nearby droplets, forming a new larger droplet. This is quite different from Ostwald Ripening, because it does not involve by transport of species from small droplets to large droplets.

During the coalescence stage one expects stepwise growth for the maximum droplet size $D_{\max }$. We measured the size of the largest droplet on several micrographs for different $\mathrm{BN}$ samples oxidized for 17 time periods ranging from $0.5-30 \mathrm{~h}$. The $D_{\max }$ values are also shown in Fig. 2, for the largest droplet that was observed in the field of view of each micrograph (about 15 $\mathrm{mm}^{2}$ ). These have considerable scatter, but can be plausibly represented as stepwise growth with two prominent steps. The lines fit to through these points were drawn with the same scaling exponent as the $\langle D\rangle$, following Beysens and Knobler. ${ }^{2}$ The first step represents about a doubling of the maximum droplet size, but the second step larger by a factor of about 4.5.

Unlike continuous condensation, the oxidation of $\mathrm{BN}$ at this temperature involves a combination of the formation of fresh liquid $\mathrm{B}_{2} \mathrm{O}_{3}$ by reaction with $\mathrm{BN}$, and the loss of $\mathrm{B}_{2} \mathrm{O}_{3}$ by evaporation, as the overall process results in a weight loss. At $1200^{\circ} \mathrm{C}$, weight loss is roughly linear with time at about $0.4 \% / \mathrm{h}$, so the liquid droplet pattern develops under a dynamic balance between formation of fresh liquid oxide and evaporation of existing liquid. It appears that throughout this process, the distribution of average and maximum liquid droplet size is governed by breath figure kinetics.

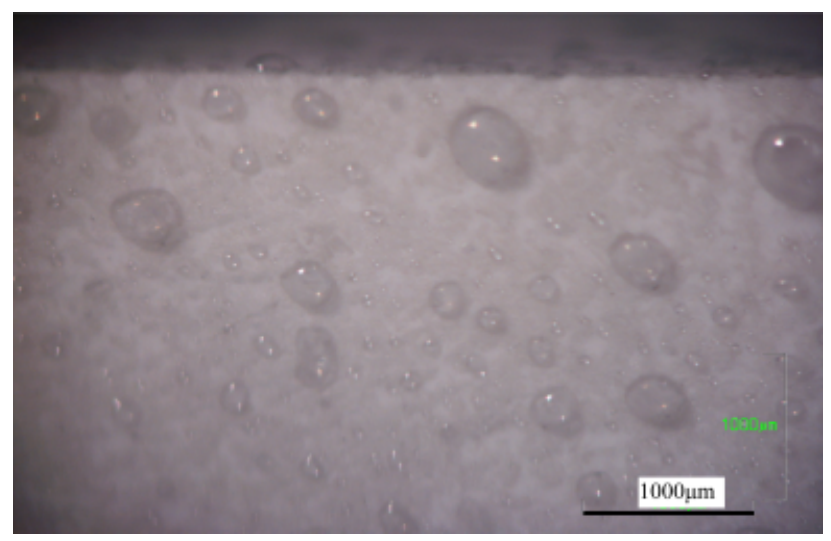

Manuscript No. 24436. Received March 18, 2008; approved March 25, 2008 The authors thank the Vietnam Educational Foundation and the Office of Naval Research for financial support under the grant N00014-02-1-0034.

Author to whom correspondence should be addressed. peterjon@umich.edu
Fig. 1. Glassy $\mathrm{B}_{2} \mathrm{O}_{3}$ droplets on the surface of boron nitride oxidized in dry air for $30 \mathrm{~h}$ at $1200^{\circ} \mathrm{C}$. 


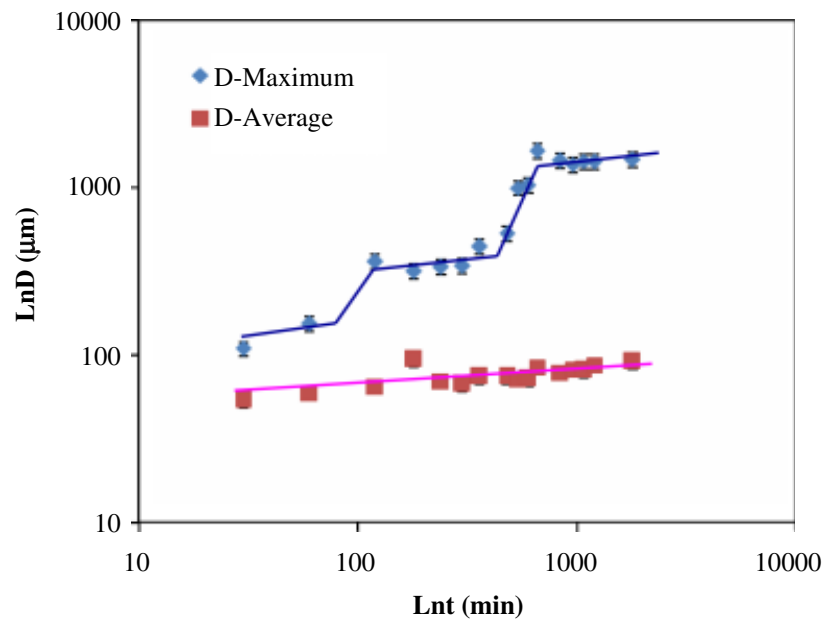

Fig. 2. Average droplet diameter $\langle D\rangle$ with regression line $t^{0.9}$ and maximum droplet diameter with two steps $D_{\max }$.

\section{References}

${ }^{1}$ L. Rayleigh, "Breath Figures," Nature, 86 [2169] 416-7 (1911).

${ }^{2}$ D. Beysens and C. M. Knobler, "Growth of Breath Figures," Phys. Rev. Lett., 57 [12] $1433(1986)$

${ }^{3}$ J.-L. Viovy, D. Beysens, and C. M. Knobler, "Scaling Descriptions for the Growth of Condensation Patterns," Phys. Rev. A, 37 [12] 4965-70 (1988).

${ }^{4}$ M. Srinivasarao, D. Collings, A. Phillips, and S. Patel, "Three-Dimensionally Ordered Array of Air Bubbles in a Polymer Film," Science, 292, 79-83 (2001).

${ }^{5}$ D. Beysens, "The Formation of Dew," Atmos. Res., 39, 215-37 (1995).

${ }^{6} \mathrm{M}$. Haupt, S. Miller, R. Sauer, K. Thonke, A. Mourran, and M. Moeller, "Breath Figures: Self-Organizing Masks for the Fabrication of Photonic Crystals and Dichroic Filters," J. Appl. Phys., 96 [6] 3065 (2004).

${ }^{7}$ A. E. Saunders, J. L. Dickson, S. P. Shah, M. Y. Lee, K. T. Lim, K.P. Johnston, and B. A. Korgel, "Breath Figure Templated Self-Assembly of Porous Diblock Copolymer Films," Phys. Rev. E, 73, 031608 (2006).

${ }^{8}$ N. Jacobson, S. Farmer, A. Moore, and H. Sayir, "Oxidation of Boron Nitride: I, Monolithic Boron Nitride," J. Am. Ceram. Soc., 82 [2] 393-8 (1999).

${ }^{9}$ T. B. Do and J. W. Halloran, "Influence of Yttria, Alumina, and Silica on the Oxidation of Boron Nitride," J. Am. Ceram. Soc., (2008), Submitted.

${ }^{10}$ A. Steyer, P. Guenon, D. Beysens, and C. M. Knobler, "Two-Dimensional Ordering During Droplet Growth on a Liquid Surface," Phys. Rev. B, 42 [1] 1085-9 (1990). 